\title{
Nanoclustering of vacancies in thin metal films revealed by x-ray diffuse scattering
}

\author{
Chinkyo Kim \\ Department of Physics and Research Institute for Basic Sciences, Kyung Hee University, 1 Hoegi-dong, \\ Dongdaemoon-gu, Seoul 130-701, Korea \\ Rui Feng and Edward H. Conrad \\ School of Physics, Georgia Institute of Technology, Atlanta, Georgia 30332 \\ Paul F. Micelia) \\ Department of Physics and Astronomy, University of Missouri at Columbia, Columbia, Missouri 65211
}

(Received 10 July 2007; accepted 12 August 2007; published online 30 August 2007)

\begin{abstract}
The authors report the incorporation of unexpectedly large vacancy clusters into homoepitaxial $\operatorname{Ag}(001)$ films. These results, which are for a simple noble metal system, have important implications for understanding the atomic-scale kinetics of surfaces where current models have mostly ignored the role of vacancies. For films grown at $150 \mathrm{~K}$, an average vacancy cluster exhibits a local dilatation volume of $750 \AA^{3}$, which leads to a $1 \%$ compressive strain of the film. Vacancy clusters are observed even for films grown near room temperature. These in situ diffuse x-ray scattering experiments measure the local deformation around the cluster and, therefore, provide conclusive evidence of vacancy clusters. (c) 2007 American Institute of Physics.
\end{abstract}

[DOI: $10.1063 / 1.2779097]$

The interaction of an atom with clusters of other atoms and with crystalline steps on a surface forms the basic building blocks for the assembly of condensed matter. Thus, the evolving morphology of nanostructures and thin films during their growth or self-assembly is a valuable laboratory for understanding the underlying atomic-scale mechanisms.

There has been a considerable amount of work investigating how atomic-scale processes conspire to create particular structures. ${ }^{1}$ Increasingly, sophisticated simulations can explore the relative importance of different atomic-scale mechanisms that lead to specific surface morphologies. 1,2 These simulations, however, require insight from experimental work in order to establish the rates or even the relevance of different kinetic processes. As will be shown in this letter, vacancy formation is an important mechanism that has been overlooked, even in simple noble metal homoepitaxial systems that were thought to be well understood.

Most in situ experimental studies of film growth are performed by surface-sensitive techniques that probe only the surface and, thus, there has been little experimental or theoretical investigation into the role of the subsurface. However, without a working knowledge of what happens below the surface, a critically important part of the story is missing. For example, it is well known that in heteroepitaxial systems (films on dissimilar substrate material) the strain arising from the substrate-film interface can dramatically affect the growth. This can lead to island formation on top of a thin wetting layer (Stranski-Krastanov growth), ${ }^{3}$ it can also alter kinetic barriers such as the barrier for diffusion over crystalline step edges, ${ }^{4}$ or it can lead to pattern formation. ${ }^{5}$

In this letter, we present the results of in situ x-ray scattering experiments that reveal unexpectedly large vacancy clusters incorporated into a noble metal film during homoepitaxial growth (films on identical substrate material).

${ }^{\text {a)}}$ Electronic mail: micelip@missouri.edu
Thus, despite the high adatom mobility on the surface, there are atomic-scale kinetic pathways that not only permit the initiation of vacancy structures but also enable their enlargement and ultimately their burial as large vacancy clusters.

This has a number of important implications. First, it suggests that the kinetic processes at crystalline step edges are significantly more complex than what is assumed in currently accepted models, which prohibit vacancy formation. Moreover, since our experiments were performed on a conceptually simple system, it is anticipated that vacancy cluster incorporation will occur ubiquitously, including more complex systems where it might be harder to observe experimentally. Finally, the large strain field arising from these buried clusters is anticipated to affect physical properties. For example, it could affect transport properties or magnetic ordering as well as influence the kinetics at the surface itself, thereby providing feedback to the growth front.

The vacancy cluster size can be determined from x-ray diffuse scattering that measures the local elastic lattice distortion caused by a vacancy cluster. ${ }^{6}$ While it is possible to use other diffraction methods to measure the long-range macroscopic volume change (known as homogeneous deformation) of the crystal that will occur concomitantly with this local strain, such homogeneous deformation can also arise from interfacial forces that are unrelated to vacancy defects. Thus, these $\mathrm{x}$-ray diffuse scattering measurements provide conclusive evidence of vacancy incorporation. Our previous $\mathrm{X}$-ray reflectivity studies on homoepitaxially grown noble metal films ${ }^{7}$ found a homogeneous compressive strain that was attributed to vacancies because of the absence of a true buried interface in a homoepitaxial system. Obviously, those measurements of homogeneous deformation were unable to address the size of the vacancy cluster.

The surface preparation, growth, and x-ray scattering measurements were carried out in ultra high vacuum $\left(10^{-10}\right.$ Torr) using the in situ surface scattering instrument at the 6ID-C $\mu$ CAT beamline located at the Advanced Photon 

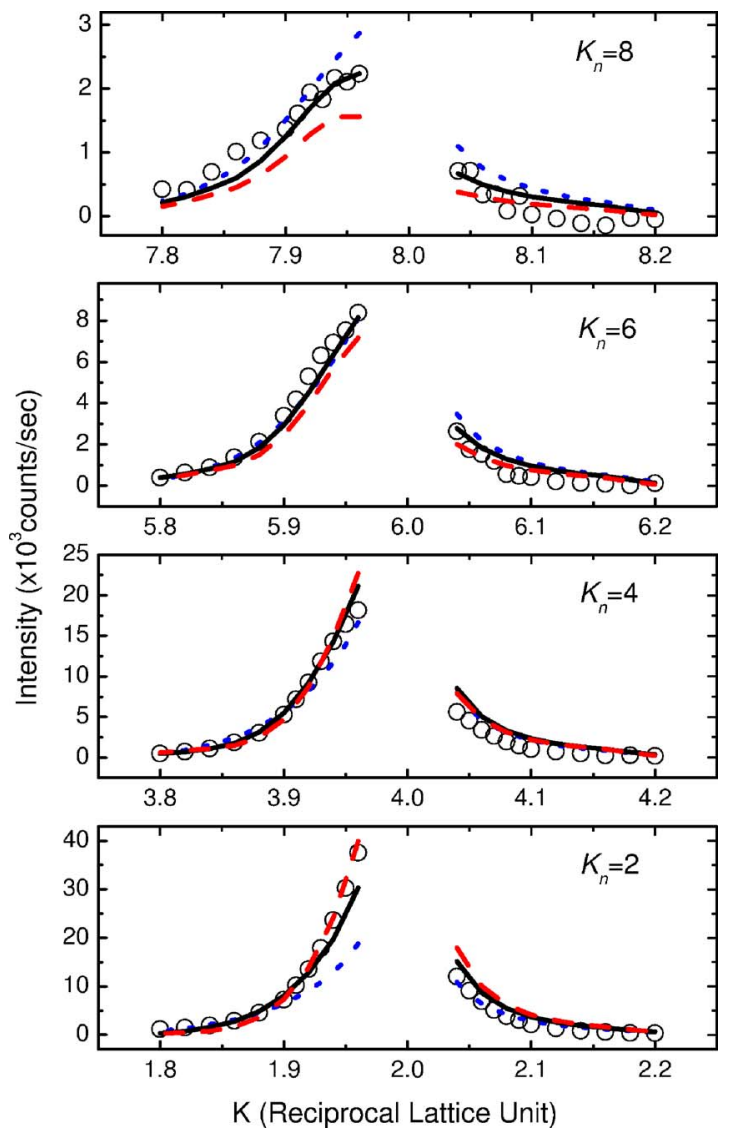

FIG. 1. (Color online) $150 \mathrm{~K}$ background-subtracted data (symbols) and model (lines) for the diffuse intensity are compared in the vicinity of four Bragg reflections with $K_{n}=2,4,6,8$. The model is calculated for $b=30,60$, and $100 \AA^{3}$, shown by the dotted, solid, and dashed lines, respectively. Because the magnitude of the diffuse intensity, its specific line shape, and its evolution with $K_{n}$ were found to depend very sensitively on the product of $b c$ (which is related to the total vacancy volume), the plots are made for $b c=0.03$ held constant. For each of the curves, $\chi^{2}$ is determined to be 700 , 480,510 , respectively, where $\chi^{2}$ is taken as a difference-squared sum of the data and model.

Source. The clean surface was prepared by repeated cycles of ion sputtering and high-temperature annealing. Auger electron spectroscopy showed no trace of contaminants. Ag films were thermally evaporated at a rate of $3.2 \mathrm{ML} / \mathrm{min}$ onto a $\operatorname{Ag}(001)$ substrate held at a fixed temperature of 150 or $285 \mathrm{~K}( \pm 10)$. Using a photon energy of $16.2 \mathrm{keV}$, scans were taken in the vicinity of Bragg reflections $(H, K, L)$ along $[0, K, 0.1]$ near $K \sim K_{n}=2,4,6,8$, where $L$ is oriented along the surface normal. The x-ray penetration length for this condition is $2500 \AA$, which is significantly more than the film thickness. The detector slit was open to $2 \mathrm{~cm}$ along the surface normal direction to allow the integration of scattered intensity. Measurements were performed before and after $100 \mathrm{ML}$ of $\mathrm{Ag}$ were deposited and the two measurements were subtracted in order to obtain the diffuse scattering from defects within the film.

The background-subtracted $150 \mathrm{~K}$ data for the four reflections are shown in Fig. 1. There is significant diffuse intensity with a pronounced asymmetry toward the low $K$ side of the Bragg position. Such asymmetry is a well-known signature of vacancies and it arises from the additional space around the vacancy. ${ }^{6}$ The strong decrease of diffuse intensity with increasing $K_{n}$ indicates a large static Debye-Waller atDownloaded 27 May 2010 to 128.206.162.204. Redistribution subje tenuation which suggests vacancy clusters that are significantly larger than monovacancies.

The cluster size can be estimated from these data through a calculation of the diffuse scattering profiles. Physically, the diffuse scattering comes from the local elastic strain field arising from the vacancy cluster; the contrast scattering from the missing atoms is negligible. A scattering theory from defects in thin films has been presented by Barabash and Krivoglaz ${ }^{8}$ from which it can be shown ${ }^{8,9}$ that a simpler model by Dederichs ${ }^{6}$ is applicable when the interfaces are negligible: when $q_{\|} D \gg 1$, where $D$ is the film thickness and $q_{\|}$is the difference of the wave vector transfer from the Bragg position, projected into the film plane. This limit applies to our experimental conditions and a complete description of our analysis using the Dederichs model will appear elsewhere. ${ }^{9}$ There are just two free parameters: the concentration of vacancy clusters $c$ and the strength of the dilatation $b$ represented by an isotropic elastic displacement field $\mathbf{t}(\mathbf{r})=-b\left(\mathbf{r} / r^{3}\right)$. This spherical vacancy cluster causes a negative volume dilatation, ${ }^{10} \Delta v_{v}=4 \pi b$.

A comparison of the experimental data with the numerical calculations is shown in Fig. 1. The best fit to the data gives $b=60 \pm 15 \AA^{3}$ and $c=0.0005 \pm 0.0002$. This implies that $\Delta v_{v}=750 \AA^{3}$, which would conservatively estimate 50 missing atoms per cluster if one equates the dilatation volume to the actual volume of missing atoms. However, the (negative) dilatation volume is known to be less than half of the atomic volume $\Omega$ for monovacancies in noble metals, ${ }^{11}$ thus suggesting at least twice this number of missing atoms. Because the dilatation will likely saturate with increasing cluster size, it is conceivable that the number of missing atoms in a cluster would be considerably larger than this. Finally, the measured $\Delta v_{v}$ is an average value and, evidently, its size distribution is not too broad since the model calculation represents the intensity, width, and asymmetry of the data quite well for each $K_{n}$. It should be emphasized that utilizing the diffuse intensity from four Bragg reflections, rather than from a single one, greatly improves the sensitivity of these measurements; it places a stringent constraint on the estimate of $\Delta v_{v}$ and $c$.

The discovery of large vacancy clusters also explains the origin of the unusually large homogeneous strain found in the previous $\mathrm{x}$-ray reflectivity studies. ${ }^{7}$ The homogeneous volume change of the crystal is related to the local volume dilatation from the clusters according to, ${ }^{10} \Delta V / V=-3[(1$ $-\sigma) /(1+\sigma)]\left(\Delta v_{v} / \Omega\right) c$, where $\sigma=0.37$ is the Poisson ratio for $\mathrm{Ag}$. From the above estimate of $\Delta v_{v}$ and $c$, we find $\Delta V / V=-0.03$, which agrees well with the observed ${ }^{7}$ linear homogeneous strain $\Delta d / d=-0.01$.

It is interesting that we also observe vacancy clusters near room temperature. Although there was insufficient signal at large $K_{n}$ to perform a similar analysis, Fig. 2 compares data at 150 and $285 \mathrm{~K}$ for the $K_{n}=4$ reflection. The roughly ten-fold decrease in intensity suggests a similar decrease in the vacancy concentration (assuming no change in cluster size), which demonstrates that a significant concentration of vacancy clusters is present even for films grown near room temperature.

Without experimental data to motivate them, theoretical models of film growth have largely been constructed to eliminate the incorporation of vacancies. The key construct used to eliminate vacancies is "downward funneling," which requires atoms deposited at step edges to move to the lower to AlP license or copyright; see http://apl.aip.org/apl/copyright.jsp 


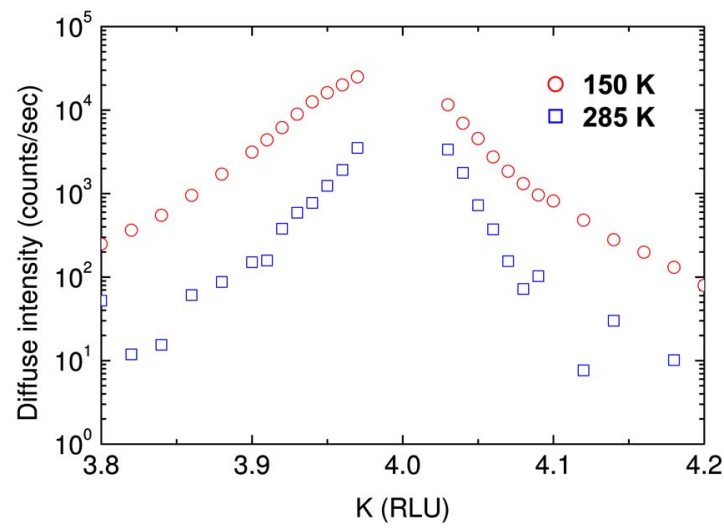

FIG. 2. Comparison of the background-subtracted diffuse scattering intensity for $\mathrm{L}=0.02$ near $K_{n}=4$ for $100 \mathrm{ML}$ films grown at two temperatures, 150 and $285 \mathrm{~K}$. The data shows that vacancy clusters are present even for films grown near room temperature.

terrace level into sites having a higher coordination. ${ }^{12}$ Its smoothing effect competes with the Ehrlich-Schwoebel stepedge diffusion barrier ${ }^{13}$ that causes a roughening of the surface and it has been quite successful in quantitatively describing the evolution of surface morphology during homoepitaxial crystal growth. ${ }^{1}$ It was also used to explain the reentrant smooth growth that was observed experimentally at low temperature $(\sim 200 \mathrm{~K})$ on $\mathrm{Cu}(001)$ (Ref. 14) and $\mathrm{Ag}$ (001) (Ref. 15) surfaces where the increased step density at low temperatures allows enhanced smoothing through downward funneling. ${ }^{15}$ At even lower temperatures $<50 \mathrm{~K}$, $\mathrm{Ag}(001)$ exhibits reentrant rough growth that has been modeled by restricting the downward funneling mechanism. ${ }^{16} \mathrm{Al}-$ though this enables the formation of vacancies, restricted funneling in the higher temperature range of our experiments would lead to surfaces that are much rougher than observed experimentally and, therefore, inconsistent with known kinetics. Indeed, homoepitaxial $\mathrm{Ag}(001)$ films grow layer by layer at room temperature. ${ }^{17}$ Moreover, the vacancy clusters that we observe at these higher temperatures are quite large. These facts bring into question how the vacancies actually form. Since the funneling mechanisms are a geometrical construction, it is likely that more complex physical processes are at work. For example, there could be collective processes involving several atoms that stabilize structures at step edges that permit the formation of vacancy clusters. Theoretical investigation into these mechanisms would require multiscale or molecular dynamics simulations involving a significant number of atoms. ${ }^{18,19}$

Finally, we emphasize that the strain fields generated by these large vacancy clusters could be very important. Indeed, the local strain fields are large enough to allow their detection by x-ray scattering. Not only could the strain fields influence physical properties such as electrical conductivity, ${ }^{20}$ but they could also influence the kinetics at the surface. For example, strain is known to reduce the step-edge diffusion barrier for $\operatorname{Ag}(111)$ (Ref. 4) and it could locally change nucleation rates. Thus, once incorporated, vacancy clusters could significantly alter the kinetics at the growth front.
In conclusion, our $\mathrm{x}$-ray diffuse scattering measurements of the local strain fields provide conclusive evidence of vacancy clusters in homoepitaxially grown Ag films. These results, which are for a relatively simple and supposedly wellunderstood noble metal system, have important fundamental implications for the kinetics of film growth. With a typical dilatation volume of $750 \AA^{3}$, the large vacancy clusters observed at relatively high temperatures call for a better understanding of the atomic-scale mechanisms that control the growth morphology of films.

Financial support is gratefully acknowledged from the University of Missouri Research Board, the National Science Foundation under Grant No. DMR0706278, the Petroleum Research Fund under Grant No. 41792-AC10 (P.F.M. and C.K.), the Canim Scientific Group (E.H.C. and R.F.), and the Seoul Research and Business Development Program under Grant No. 10583 (C.K.). The Advanced Photon Source is supported by the DOE Office of Basic Energy Sciences under Contract No. W-31-109-Eng-38. The $\mu$ CAT beam line is supported through Ames Laboratory, operated for the U.S. DOE by Iowa State University under Contract No. W-7405Eng-82.

${ }^{1}$ For a review, see J. W. Evans, P. A. Thiel, and M. C. Bartelt, Surf. Sci. Rep. 61, 1 (2006).

${ }^{2}$ Y. Shim and J. G. Amar, Phys. Rev. B 73, 035423 (2006).

${ }^{3}$ M. Zinke-Allmang, L. C. Feldman, and M. H. Grabow, Surf. Sci. Rep. 16, 377 (1992).

${ }^{4}$ K. Bromann, H. Brune, H. Roder, and K. Kern, Phys. Rev. Lett. 75, 677 (1995).

${ }^{5}$ R. Plass, J. A. Last, N. C. Bartelt, and G. I. Kellogg, Nature (London) 412, 875 (2001).

${ }^{6}$ P. H. Dederichs, Phys. Rev. B 4, 1041 (1971).

${ }^{7}$ C. E. Botez, K. Li, E. D. Lu, W. C. Elliott, P. F. Miceli, E. H. Conrad, and P. W. Stephens, Appl. Phys. Lett. 81, 4718 (2002); C. E. Botez, W. C. Elliott, P. F. Miceli, and P. W. Stephens, Phys. Rev. B 66, 075418 (2002); C. E. Botez, P. F. Miceli, and P. W. Stephens, Phys. Rev. B 66, 195413 (2002).

${ }^{8}$ R. I. Barabash and M. A. Krivoglaz, Sov. Phys. Solid State 29, 1768 (1987); M. A. Krivoglaz, X-ray and Neutron Diffraction in Nonideal Crystals (Springer, New York, 1996).

${ }^{9}$ C. Kim, R. Feng, E. H. Conrad, and P. F. Miceli (unpublished).

${ }^{10}$ J. D. Eshelby, J. Appl. Phys. 25, 255 (1954).

${ }^{11}$ C. W. Tucker and J. B. Sampson, Acta Metall. 2, 433 (1954); P. Ehrhart, K. H. Robrock, and H. R. Schober, in Physics of Radiation Effects in Crystals, edited by R. A. Johnson and A. N. Orlov (Elsevier, New York, 1986).

${ }^{12}$ J. W. Evans, D. E. Sanders, P. A. Thiel, and A. E. DePristo, Phys. Rev. B 41, R5410 (1990).

${ }^{13}$ G. Ehrlich and F. G. Hudda, J. Chem. Phys. 44, 1039 (1966); R. L. Schwoebel, J. Appl. Phys. 40, 614 (1969).

${ }^{14}$ C. E. Botez, P. F. Miceli, and P. W. Stephens, Phys. Rev. B 64, 125427 (2001).

${ }^{15}$ C. R. Stoldt, K. J. Caspersen, M. C. Bartelt, C. J. Jenks, and J. W. Evans, Phys. Rev. Lett. 85, 800 (2000).

${ }^{16}$ K. J. Caspersen and J. W. Evans, Phys. Rev. B 64, 075401 (2001).

${ }^{17}$ W. C. Elliott, P. F. Miceli, T. Tse, and P. W. Stephens, Phys. Rev. B 54, 17938 (1996).

${ }^{18}$ O. Trushin, A. Karim, A. Kara, and T. S. Rahman, Phys. Rev. B 72, 115401 (2005)

${ }^{19}$ Y. Shim and J. G. Amar, Phys. Rev. B 71, 115436 (2005).

${ }^{20}$ S. B. Arnason, S. P. Hershfield, and A. F. Hebard, Phys. Rev. Lett. 81, 3936 (1998). 